\title{
ACOUSTIC CHARACTERISTICS OF NOISE ABSORBING BARRIERS
}

\author{
Gleb Nazarov, Dmitry Nepryakhin, and Alexandr Komkin* \\ B M STU, Power Engineering Department, 105005, M oscow, Russia
}

\begin{abstract}
The acoustic characteristics of the semi-infinite noise barrier were studied using finite element modeling in the COM SOL Multiphysics software package. The features of the finite element partition into the accuracy of the results are estimated. The results of numerical calculations of the acoustic efficiency of a barrier with and without a sound absorbing layer are presented. The influence on the acoustic efficiency of the noise barrier of the length and thickness of the sound absorbing layer is analyzed.
\end{abstract}

\section{Introduction}

Currently, one of the most effective means of dealing with noise in a habitat is noise acoustic barriers. They are designed to protect the population from harmful sound effects coming from highways and railways, construction sites, industrial equipment, and other noise origins. Such barriers vary in purpose, shape, and material for their manufacture. In designing a noise barrier, the terrain features and characteristics of noise origin should be considered.

Calculation and design of acoustic barriers both in our country and abroad are paid great attention [1-15]. Moreover, as a rule, the effectiveness of acoustic barriers is estimated using the Maekawa formula [1-3], which is the result of approximating experimental data in which the Fresnel number is the main calculation parameter. This approach to the calculation is attractive due to its simplicity, but has its limitations, for example, the fact that the acoustic barrier can only be considered absolutely rigid. And although it is very difficult to take these factors into account when calculating by analytical methods, much attention continues to be paid to the development of these methods [4-14]. At the same time, it should be borne in mind that in the near future, numerical methods will become the main tool in calculating acoustic barriers and other areas of applied acoustics [15-19]. According to [15], using finite element modeling, the features of the formation of an acoustic pattern in the vicinity of the barrier located on the underlying surface are studied and the laws of the influence of this surface on the acoustic efficiency of the barrier are analyzed.

The article considered the semi-infinite noise barrier with sound-absorbing coverage and studied the effect of such an acoustical lining on the acoustic efficiency of the barrier, using COMSOL Multiphysics software package.

* Corresponding author: akomkin@ mail.ru 


\section{Finite element model of the noise barrier}

In order to study the acoustic characteristics of the noise barrier with sound absorbing coverage and obtain an acoustic pattern around it, a two-dimensional finite element method was used. In COMSOL Multiphysics, a circle with a diameter of 70 meters was constructed with a center at the beginning of the coordinate system, in which noise source was also located (Figure 1). Following the two-dimensionality of this calculation model, noise source is linear. The boundary conditions on the circle bounding the domain under consideration correspond to the characteristic impedance of air $Z=\rho c=411,6 \mathrm{~Pa} \cdot \mathrm{s} / \mathrm{m}$, which ensures complete reflection of sound at this boundary. The model studied the symmetric arrangement of noise source (NS) and computational point (CP) relative to the barrier (Figure 1). The angle $\alpha$ formed by the horizontal coordinate axis and the line connecting the upper edge of the barrier to the location of noise source or computational point changed. The height of the semi-infinite barrier relative to the horizontal axis of the coordinate system in all cases studied remained constant $-6 \mathrm{~m}$. In this case, obviously, a change in the angle $\alpha$ led to a change in the distance of noise source and computational point to the barrier. The linear noise source during the calculations was determined by the intensity of $1 \mathrm{~mW} / \mathrm{m} 2$ at a distance of $5 \mathrm{~m}$ from origin.

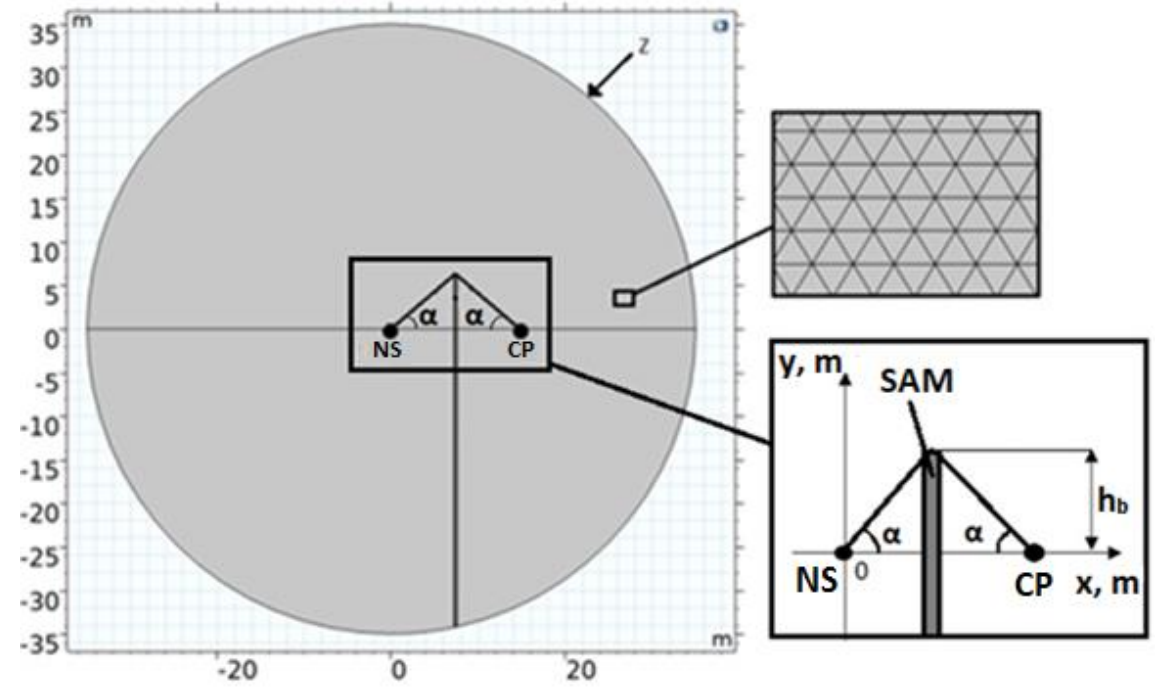

Fig. 1. Calculation finite element barrier model.

Grid generation is an important stage of finite element modeling since it directly determines the cost of computing resources during the calculation itself. Here a compromise is needed between the accuracy of the results obtained with a detailed partition and the resultant increase in the computation time. This issue has been given due attention. In modeling, non-uniform partitioning was used. With this in mind, the sound pressure level was calculated at the computational point for various maximum finite element sizes $\Delta x$. The calculations were carried out upon excitation by the point origin of sound at frequencies of $400 \mathrm{~Hz}$ and $800 \mathrm{~Hz}$. In this case, the parameter $n$ was used as the main variable, which is determined by the ratio of the sound wavelength $\lambda$ to the maximum size of the finite element: $n=\lambda / \Delta x$ and showing the number of such elements that fit on one wavelength. The reference value was the sound pressure level at the starting point at $n=5$. At the same time, the maximum finite element size $\Delta x=0.172 \mathrm{~m}$ corresponds to a frequency of $400 \mathrm{~Hz}$, and $\Delta x=0.086 \mathrm{~m}$ corresponds to a frequency of $800 \mathrm{~Hz}$. Next, the sound pressure levels were 
calculated at lower values $n$. Then the difference $\Delta L_{n}=L_{5}-L_{n}$ was determined, which can be considered as a calculation error, which leads to a coarser grid of partitions. The graphical dependencies of $\Delta L_{n}$ on $n$ for the two frequencies under consideration are presented in Figure 2 .

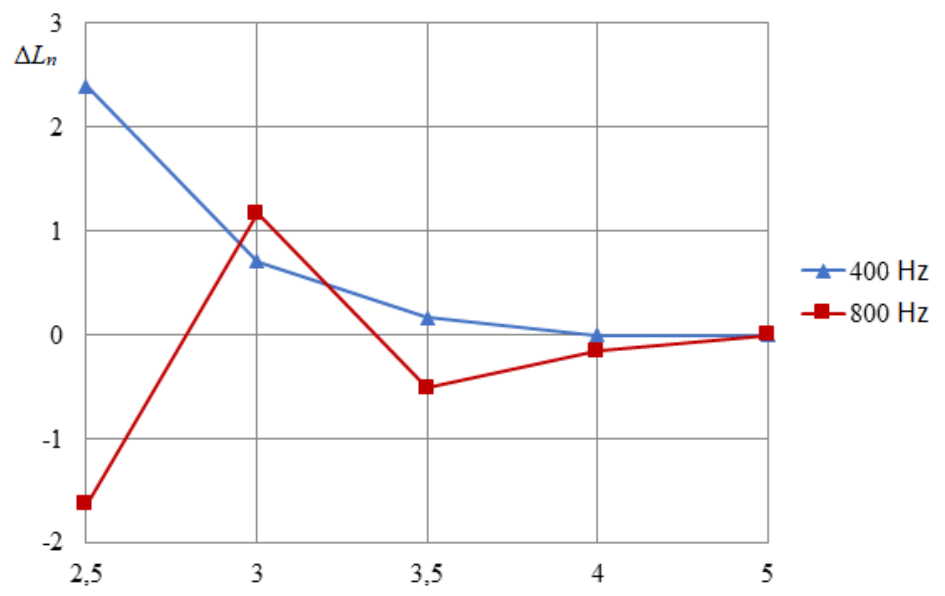

Fig. 2. Dependence of calculation error on parameter $n$.

From the presented graphs it follows that small computational errors are achieved for $\mathrm{n}>4$, i.e. when at least four finite elements of maximum size fit into the sound wavelength. Also, let us note that if for a frequency of $400 \mathrm{~Hz}$ the error increases smoothly with decreasing $\mathrm{n}$, then for a frequency of $800 \mathrm{~Hz}$ these changes are more complex, unsystematic in nature, and large errors are also typical for a higher frequency. In general, this conclusion is consistent with the results of [15], where a more accurate partition was used with a maximum finite element size of $0.01 \mathrm{~m}$. It was found that at frequencies above $4000 \mathrm{~Hz}$, the errors in the calculations become higher. The authors explained this result by the fact that, at the boundaries of the considered computational domain, the impedance boundary conditions did not fully correspond to the conditions of the characteristic impedance of air and this became critical at high frequencies.

Subsequently, when conducting numerical calculations, an uneven partition was used with the maximum size of the final element $\Delta \mathrm{x}=0.086 \mathrm{~m}$, which should have provided very small calculation errors at both frequencies considered. The total number of finite elements was 1468569 .

During modeling a barrier with a layer of sound absorbing material (SAM), the layer thickness was 0.15 meters, with a barrier thickness of 0.2 meters. The characteristics of sound absorbing material were described using the Delaney-Bazley model, the main parameters of which are the frequency of sound and the resistance to blowing of the sound absorbing layer. Glass fiber with a fiber diameter of $10 \mu \mathrm{m}$ and a density of $12 \mathrm{~kg} / \mathrm{m} 3$ was taken as SAM. The blowing resistance of such a material was $1424, \mathrm{~N} \cdot \mathrm{c} / \mathrm{m}$.

\section{Results}

At the first stage of modeling, a qualitative assessment of increasing the acoustic efficiency of the barrier in the presence of a SAM layer on its surface was made. Figures 3 and 4 show patterns of the distribution of sound pressure levels in space for a model with a semi-infinite barrier with and without a SAM layer. The calculations were performed at a frequency of $400 \mathrm{~Hz}$ and $800 \mathrm{~Hz}$ for an angle $\alpha=80^{\circ}$. 


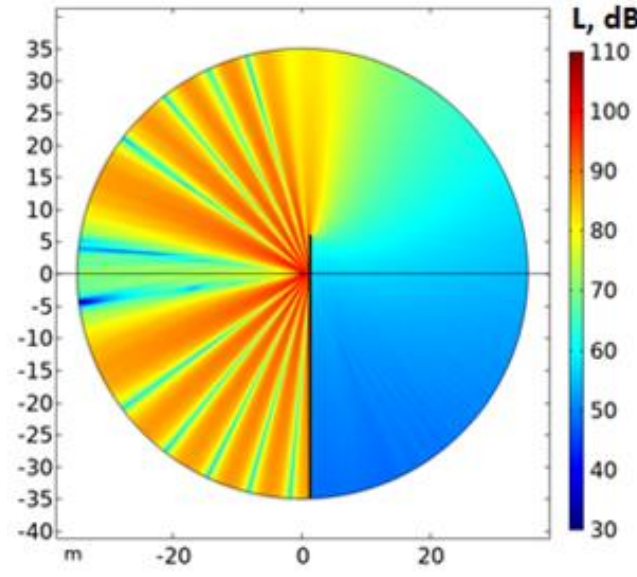

(a)

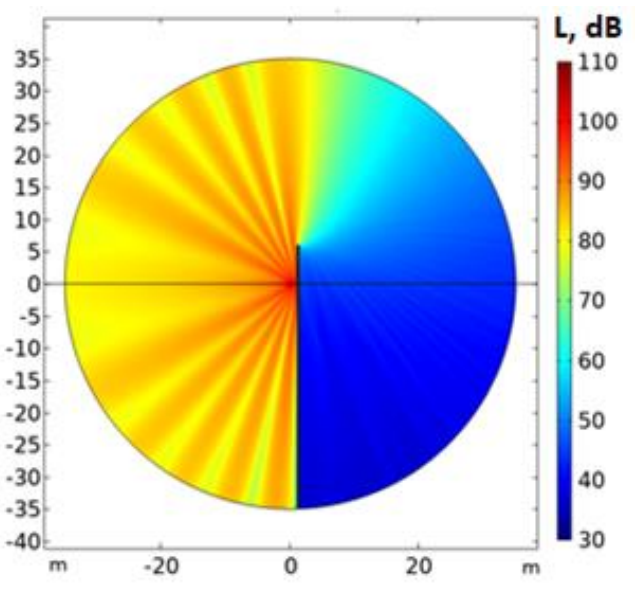

(b)

Fig. 3. Distribution pattern of sound pressure levels at a frequency of $800 \mathrm{~Hz}$ with a barrier without SAM (a) and with SAM (b).

From the consideration of Figure 3 it follows that in the space in front of the barrier, the distribution of sound pressure is a complex interference pattern due to the reflection of a sound wave from the origin on the barrier. At the same time, on the barrier with SAM, the interference pattern looks less clear, slightly smeared. In the space behind the barrier, the interference pattern is no longer observed, and the overall sound pressure level is significantly reduced, and judging by the colour palette, for the barrier with SAM this decrease is more significant and apparently amounts to about $10 \mathrm{~dB}$. This value is essentially an assessment of the effectiveness of using barrier SAM. Also, let us note that in the space above the barrier, in the first quarter of the coordinate system, between the interference domain and the domain of significant sound reduction, there is a rather narrow transition domain, let us call it diffraction domain. As follows from the patterns in Figure 3, for a barrier with SAM, the diffraction domain is narrower and, in fact, unlike a barrier without SAM, starts from the upper edge of the barrier. Moreover, for a barrier with SAM, the boundary of the diffraction domain with the domain of significant sound reduction, on the contrary, is clearer, in contrast to the interference pattern.

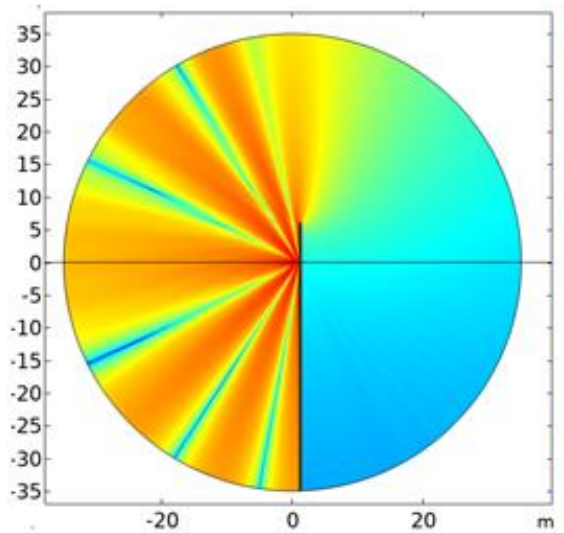

(a)

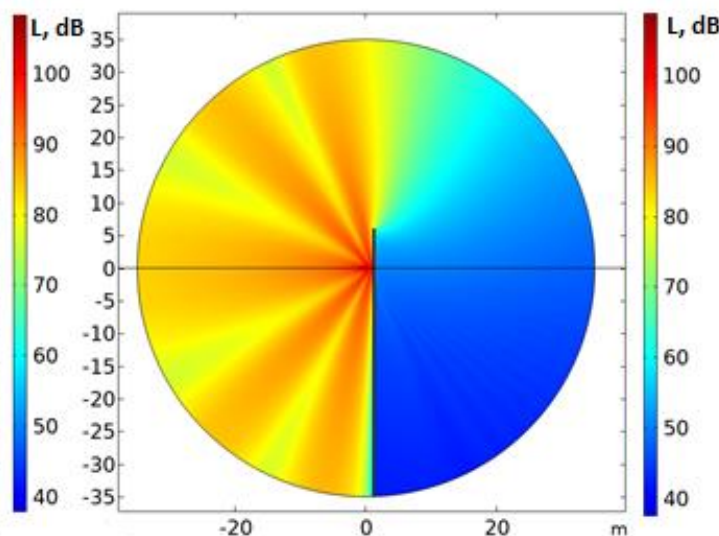

(b)

Fig. 4. Distribution pattern of sound pressure levels at a frequency of $400 \mathrm{~Hz}$ with barrier without SAM (a) and with SAM (b). 
The pattern is shown in Figure 4 for a frequency of $400 \mathrm{~Hz}$ is generally similar to that in Figure 3, but for the barrier without SAM the boundary between the diffraction zone and the zone of the acoustic shadow becomes very smeared and practically not traceable, but for the barrier with SAM it is still traceable, although slightly less distinct.

From the physical point of view, the positive effect of the presence of the SAM layer in the barrier is explained by the energy losses of the sound wave during the passage of the sound wave through this layer. As a result of this, an additional decrease in sound pressure in the space behind the barrier is observed. However, it should be noted that the detailed mechanism of this phenomenon remains unclear.

At the second stage of modeling, the effect of the length of the sound absorbing layer on the effectiveness of the acoustic barrier was investigated. This influence was estimated by the value

$$
\Delta L=L_{\text {with }}-L_{\text {without }}
$$

where $L_{\text {with }}$ - is the sound pressure level at the starting point for barrier with SAM; $L_{\text {without }}-$ is the sound pressure level at the starting point for barrier without SAM. The length of the sound absorbing layer $h$ was counted from the upper edge of the barrier and changed in $0.25 \mathrm{~m}$ incrementally.

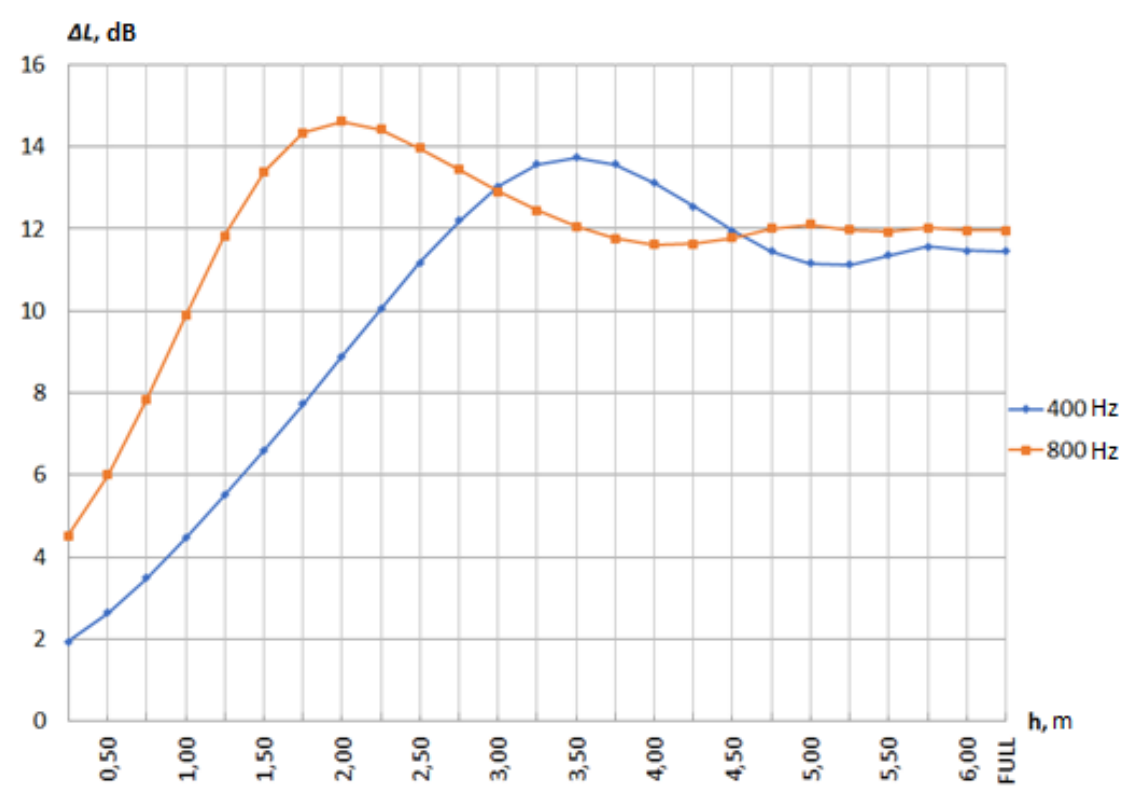

Fig. 5. Influence of sound absorbing layer length on efficiency of acoustic barrier for angle $\alpha=80^{\circ}$.

The graphs presented in Figure 5 show that the efficiency of placing a SAM layer on a barrier in a complex way depends on the length of this layer. It is especially worth noting here that this efficiency does not take its maximum value at all with the longest SAM layer length. In reality, there is a kind of transitional process. In the beginning, with the increase in the length of the absorbing layer, the efficiency of using the SAM layer gradually increases, and then when a certain maximum value is reached, it begins to decrease until it reaches a constant level, so that a further increase in the SAM length no longer leads to a change in its placement efficiency. In this case, the difference between the maximum and steady-state values of efficiency is about $2 \mathrm{~dB}$. Another feature of the considered dependences is that the SAM layer length, which corresponds to the maximum value of the efficiency, depends on 
the sound frequency (wavelength). For a frequency of $800 \mathrm{~Hz}$, this length is $2 \mathrm{~m}$, and for a frequency of $400 \mathrm{~Hz}$, it is almost twice as large - of the order of $3.5 \mathrm{~m}$. The established value of efficiency, as one would expect, for a frequency of $800 \mathrm{~Hz}$ is slightly higher than for a frequency of $400 \mathrm{~Hz}$, and is $12 \mathrm{~dB}$. The difference is about $0.5 \mathrm{~dB}$.

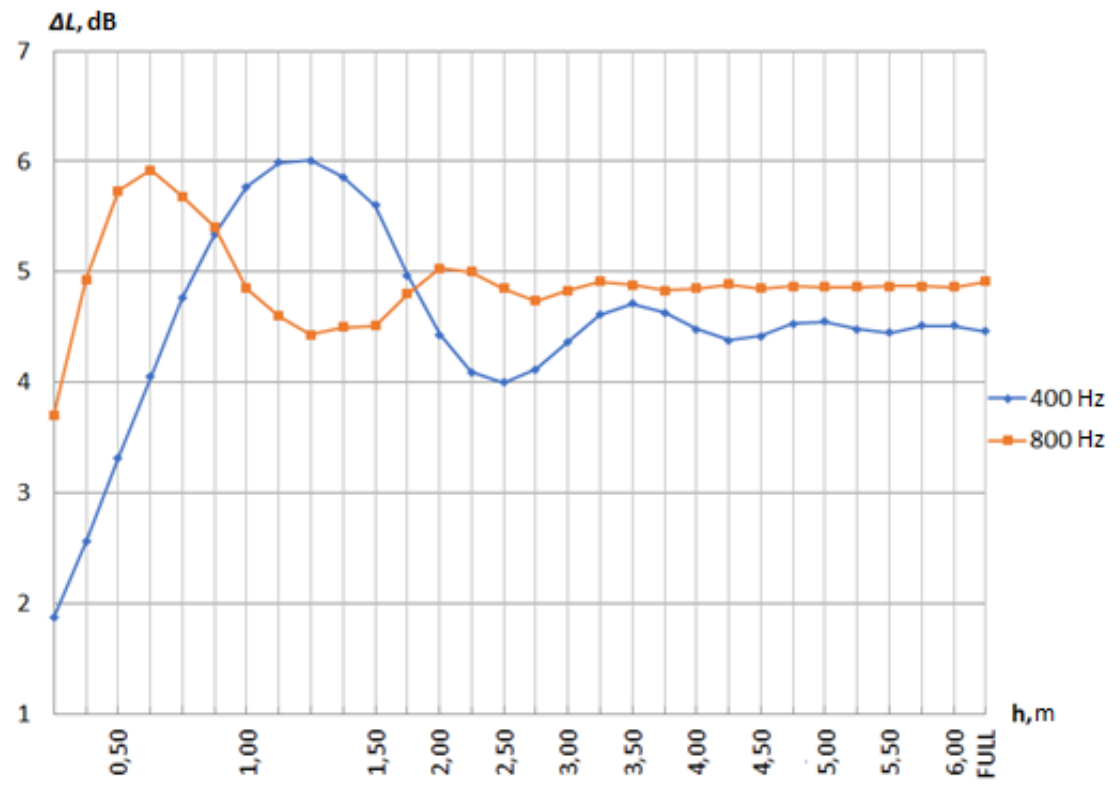

Fig. 1. Influence of sound absorbing layer length on efficiency of acoustic barrier for angle $\alpha=60^{\circ}$.

Figure 6 shows similar dependencies for the angle $\alpha=60^{\circ}$. The general nature of the change in the curves here is the same as for the angle $80^{\circ}$. The main difference is that here the SAM layer length corresponding to the maximum value decreases almost three times, making for frequencies of $800 \mathrm{~Hz}$ and $400 \mathrm{~Hz}$, respectively, $0.625 \mathrm{~m}$ and $1.2 \mathrm{~m}$. The established efficiency values also significantly decrease, to values slightly less than $5 \mathrm{~dB}$.

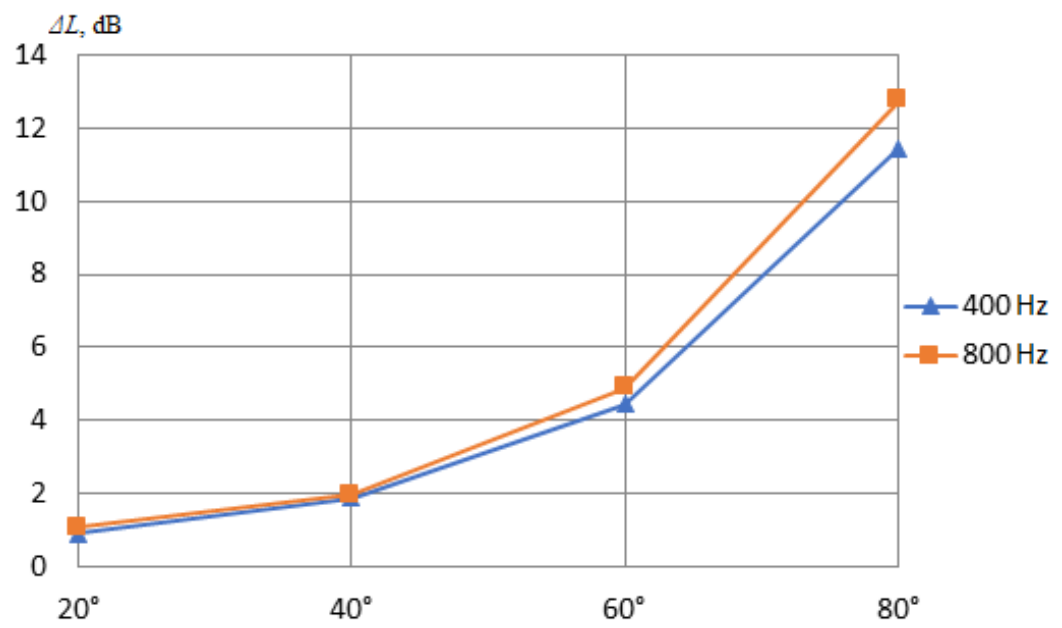

Fig. 7. Dependence of SAM layer placement efficiency on angle $\alpha$.

A general view of the dependence of the placement efficiency of the SAM layer on the acoustic barrier efficiency on the angle $\alpha$ is shown in Figure 7. These graphs show a 
significant increase in SAM efficiency in the considered range of changes in the angle $\alpha$, reaching

$10-11 \mathrm{~dB}$. Moreover, this dependence on the frequency varies slightly and is noticeable only at large values of $\alpha$.

At the third stage of the simulation, influence of thicknesses of a sound absorbing layer on the effectiveness of the noise barrier were investigated. A noise barrier with completely SAM covered surface was considered. The studied dependence for the angle $\alpha=60^{\circ}$ is presented in Figure 8.

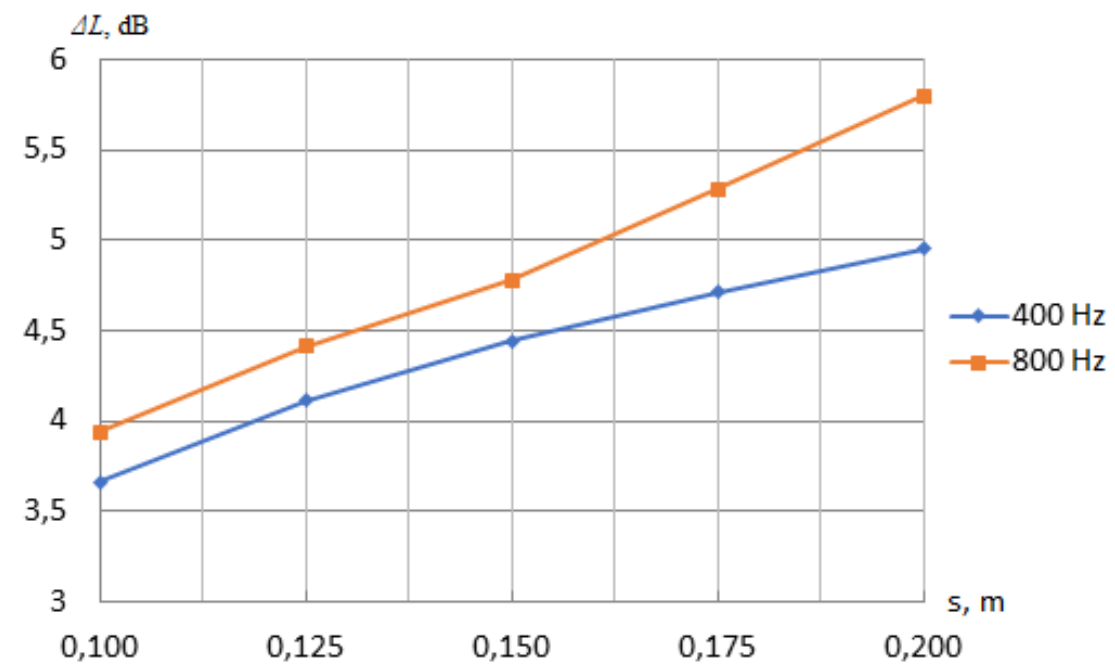

Fig. 8. Dependence of the placement efficiency of the SAM layer on its thickness.

In this case, the efficiency of placement of the SAM layer with an increase of its thickness grows monotonously enough. Besides, here the sound frequency has a noticeable effect on the acoustic efficiency of the SAM layer. With increasing frequency, it increases significantly. So, if at a frequency of $400 \mathrm{~Hz}$ with a doubling of the SAM thickness, the efficiency increases by about $1.5 \mathrm{~dB}$, then at a frequency of $800 \mathrm{~Hz}$ this increase is almost $2 \mathrm{~dB}$.

\section{Conclusion}

A finite element model of an acoustic barrier with a sound-absorbing layer developed in the COMSOL Multiphysics software and the results of numerical calculations, made it possible to establish the main laws governing the effect of a SAM layer on the surface of an acoustic barrier on its acoustic efficiency.

It was found that the efficiency of using the SAM layer on the barrier surface depends on the length of this layer. Moreover, the maximum layer efficiency does not correspond to the barrier surface completely covered by this layer, but to some optimal layer length, which is different for each frequency.

The calculations showed that the efficiency of SAM placement depends on the angle $\alpha$, substantially increasing with its increase. At the same time, this frequency is very slightly affected by the frequency of sound.

With a doubled increase in the SAM layer thickness, the SAM efficiency increases by 1.5-2 dB, and, as expected, the efficiency also increases with increasing frequency.

It is supposed that further computational studies of barriers with SAM will be carried out to study in more detail the acoustic processes that occur in barriers. 


\section{References}

1. Z. M aekawa, A ppl. A coust. 1, 157 (1968)

2. Z. M aekawa, Acoustic barriers, in Noise reduction in buildings and residential areas, Ed. by F.L. Osipov and E.Y a. Y udin. (Stroyizdat, M oscow, 1987) [in R usssian]

3. Z. M aekawa, Recent Problems with Noise Barriers, in Proceedings of the Noise-93 Conference, 31 M ay 31- 3 J une 1993, St. Petersburg, Russia (1993)

4. U. J. K urze, J. A coust. Soc. A m. 55, 504 (1974)

5. T. Isei, T. F.W. Embleton, J. E. Piercy, J. A coust. Soc. A m. 67, 46 (1980)

6. A. L (i)sperance, J. Nicolas, G. A. Daigle, J. A coust. Soc. A m. 86, 1060 (1989)

7. S. I. Hayek, A ppl. A coust. 31, 77 (1990)

8. K. Takagi, A ppl. A coust. 31, 119 (1990)

9. Y . W. Lam, A ppl. A coust. 42, 29 (1994)

10. A. M uradaliand, K. R. Fyfe, A ppl. A coust. 53, 49 (1998)

11. P. M enounou, I. J. Busch-V ishniac, D. T. Blackstock, J. A coust. Soc. A m. 107, 2973 (2000)

12. P. M enounou, J. A coust. Soc. A m. 110, 1828 (2001)

13. P. M enounou, E. S. Papaefthymiou, A ppl. A coust. 71, 351 (2010)

14. I. L. Shubin, I. E. T sukernikov, N. Nikolov, A. Pisarsky. Basics of designing noise barriers (BASTET, M oscow, 2015) [in Russsian]

15. A. I. Komkin, A. I. Bykov, A coust. Phys. 62(3), 269 (2016)

16. M. K alugin et al., Her. Bauman M osc. St. Tech. U niv. Ser. Natur. Scien. 3, 32 (2018)

17. A. E pikhin, M . K raposhin, K. V atutin The numerical simulation of compressible jet at low Reynolds number using OpenFOAM, E3S W eb of Conferences. EDP Sciences, 128, 10008 (2019)

18. S. M. Sivachev, L. L. M yagkov Thermomechanical Fatigue Analysis of Diesel Engine Piston: Finite Element Simulation and Lifetime Prediction Technique, in Proceedings of International Conference on Industrial Engineering. Springer, Cham, 109 (2019)

19. Y . I. V inogradov, G. B. M enkov, Modeling of the boundary conditions when calculating the stress-strain state of a cone-shaped aircraft nose fairing, AIP Conference Proceedings, A IP Publishing LLC, 2171. N. 1. P. 170012 (2019)

20. V. A . Kulkina, A . I. Komkin, A I. Bykov, Study of the acoustic characteristics of noise barriers, in this Conference Proceedings 